\title{
Patches of polar mesospheric summer echoes characterized from radar imaging observations with MAARSY
}

\author{
Svenja Sommer and Jorge L. Chau \\ Leibniz Institute of Atmospheric Physics at the University of Rostock, Kühlungsborn, Germany \\ Correspondence to: Svenja Sommer (sommer@iap-kborn.de)
}

Received: 27 May 2016 - Revised: 21 November 2016 - Accepted: 22 November 2016 - Published: 19 December 2016

\begin{abstract}
A recent study has hypothesized that polar mesospheric summer echoes (PMSEs) might consist mainly of localized isotropic scattering. These results have been inferred from indirect measurements. Using radar imaging with the Middle Atmosphere Alomar Radar System (MAARSY), we observed horizontal structures that support our previous findings. We observe that small-scale irregularities, causing isotropic scattering, are organized in patches. We find that patches of PMSEs, as observed by the radar, are usually smaller than $1 \mathrm{~km}$. These patches occur throughout the illuminated volume, supporting that PMSEs are caused by localized isotropic or inhomogeneous scattering. Furthermore, we show that imaging can be used to identify side lobe detections, which have a significant influence even for narrow beam observations. Improved spectra estimations are obtained by selecting the desired volume to study parameters such as spectral width and to estimate the derived energy dissipation rates. In addition, a combined wide beam experiment and radar imaging is used to resolve the radial velocity and spectral width at different volumes within the illuminated volume.
\end{abstract}

Keywords. Meteorology and atmospheric dynamics (turbulence; instruments and techniques) - radio science (remote sensing)

\section{Introduction}

Polar mesospheric summer echoes (PMSEs) are nowadays a well-understood phenomenon in the mesopause region, where turbulence plays a major role in the existence of these echoes in conjunction with charged ice particles and free electrons (Rapp and Lübken, 2004). These echoes are commonly used as tracers for wind in polar regions in the mid- dle atmosphere (e.g. Czechowsky et al., 1989; Stober et al., 2013) and are used to estimate the energy dissipation rate at mesospheric heights (Kelley et al., 1990).

Although these echoes are known since the late 1970s, some aspects of their existence, such as their aspect sensitivity (Hocking et al., 1986; Zecha et al., 2001; Chilson et al., 2002), have been explained only recently with modern, flexible radar systems. As the contradicting preceding studies regarding aspect sensitivity came to different conclusions, Sommer et al. (2016b) hypothesized that instrumental effects have to be considered together with a localized isotropic scattering mechanism. The existence of small-scale waves in the polar mesosphere is well known in noctilucent clouds (NLCs) (Baumgarten and Fritts, 2014). As NLCs and PMSEs are closely related, small-scale structures might also exist in PMSEs, which might be resolved using radar imaging. Röttger et al. (1990) concluded that PMSEs are unlikely to fill the observed volume homogeneously at $224 \mathrm{MHz}$. If these small-scale structures existed, this finding might also have impact on wind measurement techniques such as the full correlation analysis (FCA). This technique assumes a statistically homogeneous scatter (Briggs, 1968; Doviak et al., 1996; Holloway et al., 1997). Holdsworth (1995) showed that localized scattering with fewer than four scatters leads to an underestimation of the horizontal wind velocity. Here, we show that the horizontal homogeneity is not always satisfied since PMSEs are either localized or not homogeneously powered in the beam volume, similar to NLC observations. On the other hand, the observation of PMSEs on short timescales is limited by statistical effects of the scattering process. The statistical scattering properties of PMSEs on short timescales were investigated by Sommer et al. (2016a), also showing the instrumental influences on PMSE measurements. In this paper, we resolve the horizontal structure of PMSEs directly 
and discuss the observations in relation to the Sommer et al. (2016a) findings.

The observation of PMSEs depends on the antenna beam pattern and, hence, the transmitting and receiving antenna. Large aperture radars such as Middle Atmosphere Alomar Radar System (MAARSY) have a strong side lobe suppression of $-17 \mathrm{~dB}$ (Latteck et al., 2012), but PMSEs can be stronger than that, and hence they can also be detected by side lobes (Chen et al., 2008). On the other hand, wind and turbulence estimation algorithms usually assume that the received signals come from the main beam (Hocking et al., 1986), which is not necessarily the case, especially if PMSEs are not equally distributed in the observation volume and/or they are stronger than the peak-to-side lobe level. Imaging techniques such as Capon (Palmer et al., 1998; Yu et al., 2001) or maximum entropy (Hysell and Chau, 2006) are capable of resolving the scatter location within the beam volume and are able to determine spectral parameters with their dependence on incident angle (Kudeki and Sürücü, 1991). This allows us to use the information either to identify what is really coming from the main beam or to lose the side lobe information to determine neutral dynamics.

In this paper, we present studies of PMSEs with coherent radar imaging (CRI) using Capon's method. First, we show that PMSEs are composed of isotropic scattering that is horizontally inhomogeneously organized. Connected areas of isotropic scattering are here called patches. Theses patches are usually smaller than the beam volume. In the second part, we show how imaging can be used to identify side lobe detections and apply a synthetic narrow beam for spectral analysis and energy dissipation rate determination.

\section{Experimental setup and methods}

The Middle Atmosphere Alomar Radar System (MAARSY) is the only VHF $(53.5 \mathrm{MHz})$ high-power large-aperture $(866 \mathrm{~kW})$ radar in northern polar regions capable of radar imaging. Its 433 Yagi antennas, each with its own transceiver module, are combined in groups of seven in a hexagonal structure. The whole array and 15 subarrays (or 16 subarrays) can be sampled at once. To optimize the receiver configuration for radar imaging, a maximum of non-redundant baselines between all receivers is desirable. On 9 June 2015, MAARSY ran in the receiver configuration shown in Fig. 1, left side, with 145 unique baselines. The black crosses indicate possible receiver locations. However, MAARSY has a limited amount of 16 receiving channels. We chose 15 receiver locations, indicated by green circles, which are favourable for imaging, and one channel for the whole array. The visibility of the configuration is shown on the middle panel of Fig. 1 to indicate the different baseline lengths and redundant baselines. The shortest baseline in this experiment was $10.6 \mathrm{~m}$, the longest $73.3 \mathrm{~m}$. The right panel of Fig. 1 shows the antenna beam pattern of the combined 15 subar- rays used for reception, i.e. the instrument function. For further MAARSY description, see Latteck et al. (2012).

Our radar imaging experiment was complemented with a narrow-wide beam configuration, meaning that two beam sizes of 3.6 and $12.6^{\circ}$ (half power full width (HPFW)) were transmitted almost simultaneously. The interpulse period of the experiment was $4 \mu \mathrm{s}$, and we expect that PMSEs change more slowly than that; hence we expect to observe the same PMSE with two different beam sizes. The beam direction was vertical with a range resolution of $150 \mathrm{~m}$. The experiment had an interpulse period of $2 \mathrm{~ms}$ for each beam. Data were recorded after four coherent integrations, resulting in an effective time resolution of $8 \mathrm{~ms}$. Continuous $32 \mathrm{~s}$ data blocks were recorded. Spectra estimation was done with two additional coherent and four additional incoherent integrations. For further experiment details and parameters of the narrowwide beam experiment, see Sommer et al. (2016a).

For this study, the data were analysed using Capon's method (Capon, 1969; Palmer et al., 1998), as Capon's method allows the direct access to the angular resolved spectral information and is adaptive to the data, unlike the Fourier method, and is able to suppress side-lobe contribution. Capon's method is fast and simple to implement compared to other methods such as maximum entropy and yields similar results in high signal-to-noise ratio (SNR) cases (Yu et al., 2000), which usually applies for PMSEs. The angular power distribution, called brightness $B$, can be achieved by weighting each receiver signal with a linear filter to minimize side lobes adaptively and therefore possible interference. The resulting weighting vector $\boldsymbol{w}(\boldsymbol{k})$ for a certain wavenumber vector $\boldsymbol{k}=\left[\begin{array}{lll}\theta_{x} & \theta_{y} & \theta_{z}\end{array}\right]$, where $\theta_{i}$ is the direction cosine in $x, y$, and $z$ direction, respectively, can calculated as follows (Palmer et al., 1998):

$$
\boldsymbol{w}_{C}=\frac{\mathbf{V}^{-1} \boldsymbol{e}}{\boldsymbol{e}^{\dagger} \mathbf{V}^{-1} \boldsymbol{e}},
$$

where $\boldsymbol{e}^{\dagger}$ represents the conjugate transpose of matrix $\boldsymbol{e}$.

$$
\mathbf{V}=\left[\begin{array}{cccc}
V_{11} & V_{12} & \ldots & V_{1 n} \\
V_{21} & V_{22} & \ldots & V_{2 n} \\
\vdots & & \vdots & \vdots \\
V_{n 1} & V_{n 2} & \ldots & V_{n n}
\end{array}\right]
$$

is the normalized cross-correlation matrix with the elements $V_{i j}=\frac{\left\langle S_{i} S_{j}^{*}\right\rangle}{\sqrt{\left\langle\left|S_{i}\right|^{2}\right\rangle\left\langle\left|S_{j}\right|^{2}\right\rangle}}$ as the normalized cross correlation between the signals $S_{i}$ for receivers $i$ and $j$ (including also redundant baselines), and

$\boldsymbol{e}=e^{i \boldsymbol{k} \cdot \mathbf{D}_{1}} e^{i k \cdot \mathbf{D}_{2}} \ldots e^{i \boldsymbol{k} \cdot \mathbf{D}_{n}}$,

where $\mathbf{D}_{i}$ represents the centre of receiving array $i$.

The resulting brightness distribution is

$$
B_{C}(\boldsymbol{k})=\frac{1}{\boldsymbol{e}^{\dagger} \mathbf{V}^{-1} \boldsymbol{e}} .
$$



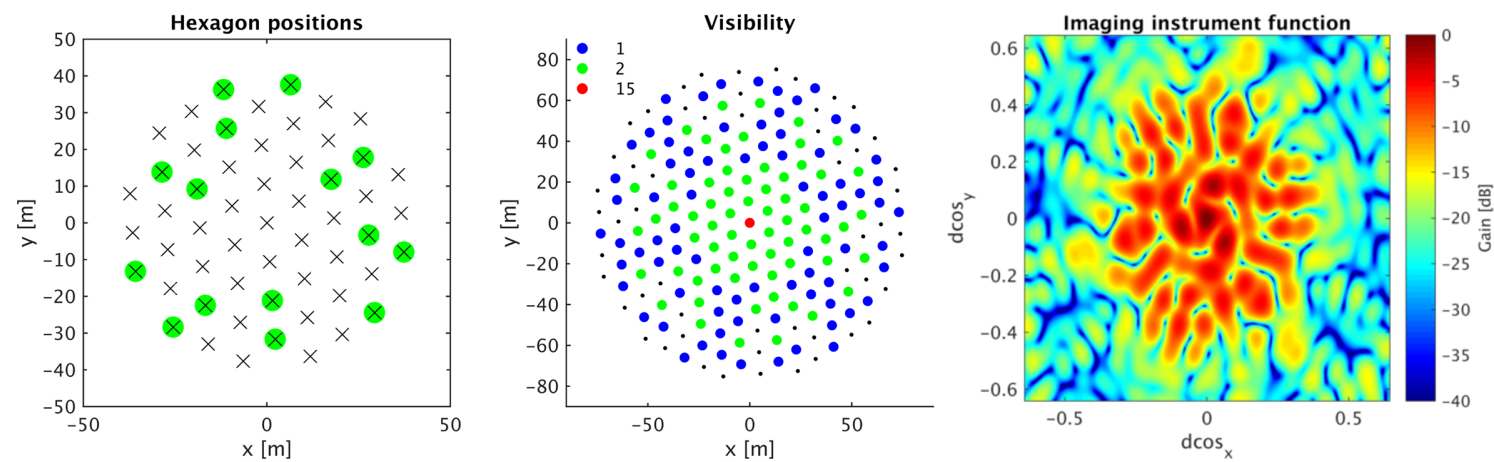

Figure 1. Left: the receiving configuration of the imaging configuration in June 2015 is indicated by green circles. All possible receiving locations are indicated by black crosses. Middle: visibility function (as referred to by astrophysicists) for the imaging configuration. All possible baselines are indicated by black dots, and the colour code is the number of redundant baselines of the configuration used for imaging. Right: instrument function of the 15 hexagons indicated in the left panel. dcos is the direction cosine.

Capon's method can be used not only for the angular power distribution but also to obtain radial velocities and spectral widths inside the beam volume, assuming quasi-stationarity during the observation period. For PMSEs, we obtain the spectrum for a certain $\boldsymbol{k}$. Hence, we apply the weighting vector, obtained with the average of the time series, on the time series signals $s$ of the $n$ receivers:

$y(t)=\boldsymbol{w}_{C}^{\dagger} \boldsymbol{s}(t)$.

The power spectral density for the parameter analysis is calculated by Fourier transforming each weighted time series for each pointing direction $\boldsymbol{k}$ and fitting a truncated Gaussian function, yielding maps for the signal, Doppler velocity shift and spectral width.

The resulting radial velocities can be used to map the wind field. A simple approach such as a Doppler beam swinging (DBS) analysis could be applied, as well as more sophisticated approaches such as volume velocity processing, allowing for inhomogeneities in the wind field (Waldteufel and Corbin, 1979).

\subsection{Results}

The signal-to-noise ratio (SNR) obtained from the narrowwide beam experiment on 9 June 2015 is shown in Fig. 2. The top panel of Fig. 2 shows the SNR of the narrow beam with a beam width of $3.6^{\circ} \mathrm{HPFW}$. The lower panel of the same figure shows the SNR of the wide beam $\left(12.6^{\circ} \mathrm{HPFW}\right)$ experiment. The detection threshold for PMSEs was a SNR of $-10 \mathrm{~dB}$. During the observation time, PMSE occurrence was almost continuous at least in the narrow beam. Comparing the results from both beams, the main features of the stronger PMSEs are observed in both beams, but the SNR of the wide beam is weaker than the SNR of the narrow beam. The most important reason is the geometry of the observations: in the wide beam experiment, the power is spread over a larger solid angle, leading to less gain at zenith. If the beam is wider, more energy will be transmitted to large off-zenith angles and scattered by PMSEs, but PMSEs appear in larger range gates at large off-zenith angles compared to a narrow beam. Decreasing the gain at zenith decreases therefore the SNR.

Due to the decreased SNR, some PMSEs cannot be detected (e.g. 07:30-08:30 UTC, $78-82 \mathrm{~km}$ ) by the wide beam but can be seen in the narrow beam. On the other hand, signal can be detected at larger ranges in the wide beam observations than in the narrow beam observations (e.g. 00:3001:00 UTC, 88-89.5 km).

The spectral parameters of the narrow beam experiment are shown in Fig. 3: (a) SNR, (b) radial Doppler velocity, (c) spectral width, (d) expected uncertainties for the Doppler velocity and (e) uncertainties for the spectral width. All the parameters are obtained from a truncated Gaussian fit like that used by Sheth et al. (2006). The red lines indicate two time intervals that are analysed later in detail with imaging. The Doppler velocity of the narrow beam varies mainly between $\pm 15 \mathrm{~m} \mathrm{~s}^{-1}$ and are quite large compared to the expected vertical wind component of only a few metres per second (Hoppe and Fritts, 1995) and indicate a horizontal wind contribution. Particularly large values $\left(>15 \mathrm{~m} \mathrm{~s}^{-1}\right)$ can be observed around 01:00 UTC above $88 \mathrm{~km}$. The spectral width is sometimes enhanced during certain periods of time, e.g. 08:30-09:00 UTC, 83 to $85 \mathrm{~km}$ and 00:30-01:00 UTC, $85 \mathrm{~km}$ and above. The enhanced spectral width at the top of PMSEs, together with the increased corresponding radial velocity, is likely due to echoes coming from antenna side lobes, as we show below.

\subsection{PMSE patch sizes}

Side lobe detection can influence the observations of PMSEs, and the illuminated volume cannot be assumed to be the expected main beam (e.g. Hocking et al., 1986). Hence, side lobe contributions have an influence on wind analysis meth- 


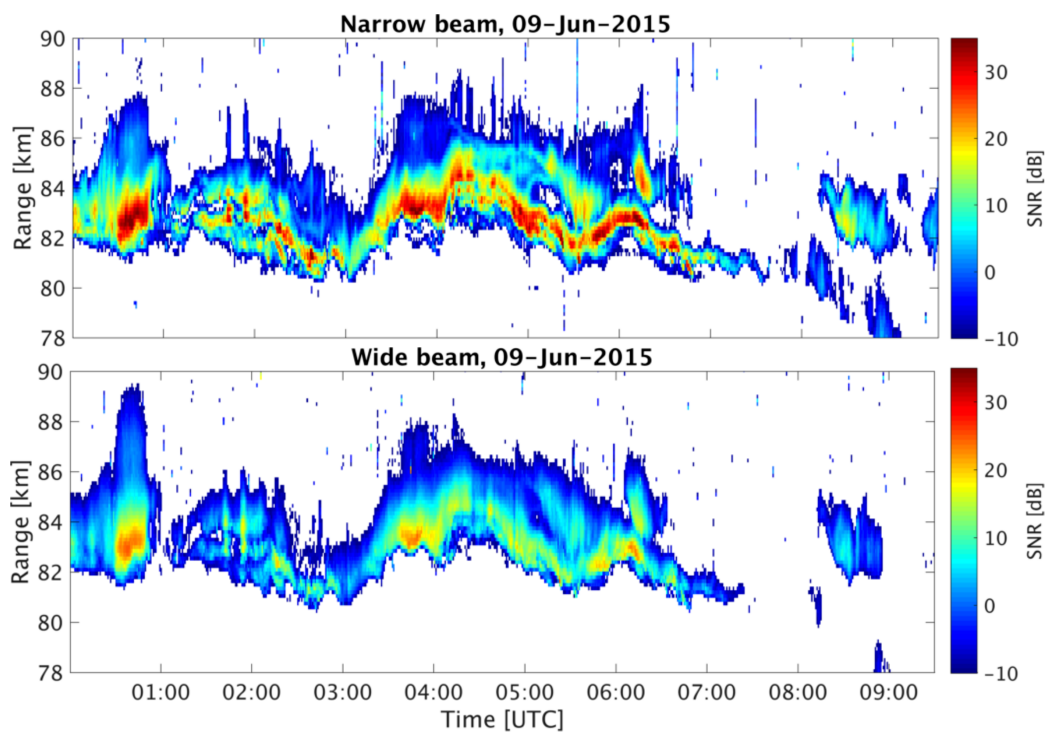

Figure 2. RTI of the SNR from (top) narrow $\left(3.6^{\circ}\right)$ and (bottom) wide $\left(12.6^{\circ}\right)$ beam of the nested beam experiment on 9 June 2015.

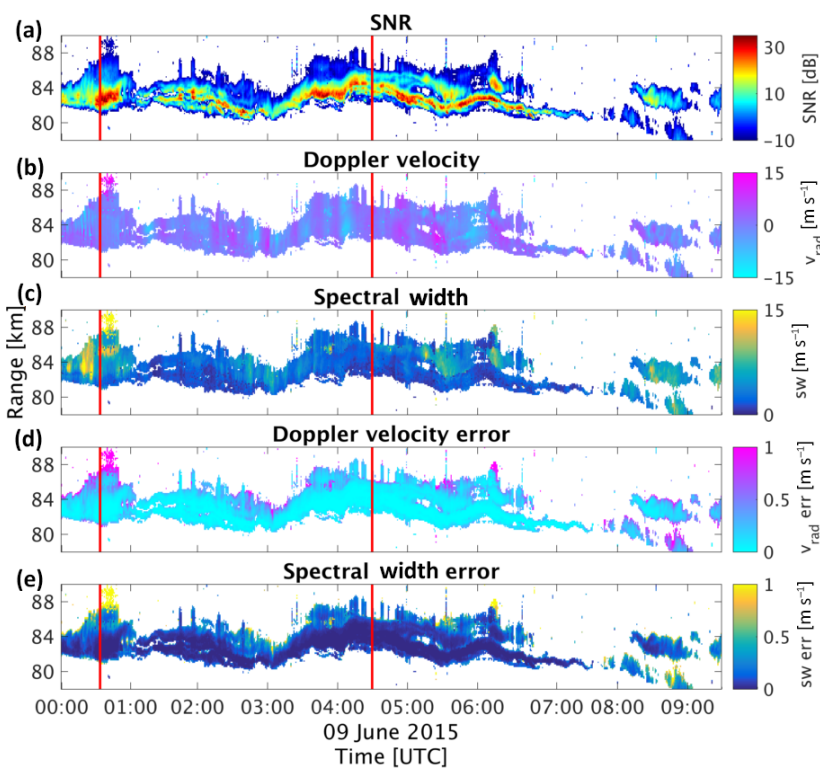

Figure 3. Analysed parameters of the narrow beam $\left(3.6^{\circ}\right)$. (a) SNR: the PMSE was strong during the observation time but got weaker in the last $2 \mathrm{~h}$. (b) Doppler velocity: the radial velocity varies between $\pm 15 \mathrm{~m} \mathrm{~s}^{-1}$. (c) The spectral width appears to be increased sometimes at the top of PMSE (00:00-01:00 UTC) or in the whole PMSE (08:00-09:00 UTC). (d) Doppler velocity error: error of the radial velocity estimation from the Gaussian fit. (e) Spectral width error: error of the width estimation from the Gaussian fit.

ods, like DBS or FCA, but these side lobe contributions could be estimated if the antenna beam pattern were known.

The remaining problem might be that the illuminated area is large and changes in PMSEs within the observed volume can occur. Therefore, using radar imaging, we analyse the sizes of PMSE patches, i.e. areas with isotropic scattering, for different beam sizes and integration times. Such patches have been hypothesized by Sommer et al. (2016b).

Figure 4 shows the obtained brightness (first row), radial velocity (second row) and spectral width (bottom row) for three adjunct altitudes after converting the image from angular space and range into Cartesian coordinates and altitude with MAARSY at $(0,0,0)$ for a $32 \mathrm{~s}$ wide beam data set. The limit for the maps was chosen to be a brightness of $25 \mathrm{~dB}$ to avoid presenting velocity and spectral width data with larger uncertainties. The white lines in the first row indicate fitted 2-D Gaussian ellipsoids. The point in time 00:33:58 UTC is marked by the first vertical red line in Fig. 3. The PMSEs were strong at the time, and the observation volume was filled with PMSEs, which can be seen in the brightness distribution. Although PMSEs occur in the whole beam volume, the strength varies. If PMSEs homogeneously filled the beam volume, the antenna beam pattern could be seen, which is not the case. In the lowest altitude, PMSEs fill almost homogeneously the beam volume while at the highest altitude displayed here, PMSEs are strongest in the upper left quadrant. In order to quantify the inhomogeneity, we fitted the peaks with $N$ 2-D Gaussian ellipsoids (following Chau and Woodman, 2001) of the form

$$
\begin{aligned}
f(x, y) & =\sum_{i=1}^{N} A_{i} \exp \left(-\left[\begin{array}{c}
x-x_{0 i} \\
y-y_{0 i}
\end{array}\right]^{\top} \mathbf{T}_{i}^{\top} \Sigma_{i}^{-1} \mathbf{T}_{i}\left[\begin{array}{l}
x-x_{0 i} \\
y-y_{0 i}
\end{array}\right]\right) \\
& +A_{N+1}
\end{aligned}
$$

with

$\Sigma_{i}=\left[\begin{array}{cc}2 \sigma_{x i} & 0 \\ 0 & 2 \sigma_{y i}\end{array}\right]$ 


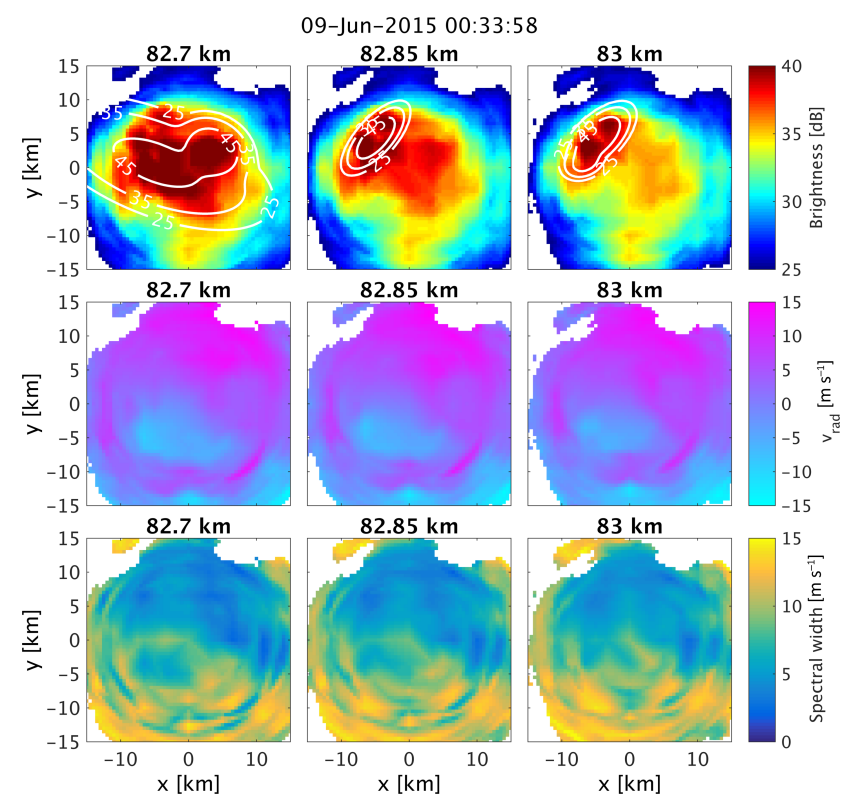

Figure 4. Spectral parameters of PMSE after converting the image to Cartesian coordinates and altitude on 9 June 2015 00:33:58 UTC. The columns show three adjunct altitudes around the strongest PMSE altitude of $82.85 \mathrm{~km}$. The brightness distributions are shown in the top row and are colour coded. The white lines indicate the fitted Gaussian ellipsoids. The middle row shows the radial velocities, indicating a horizontal wind, and the bottom row shows the imaged spectral width.

$\mathbf{T}_{i}=\left[\begin{array}{cc}\cos \theta_{i} & \sin \theta_{i} \\ -\sin \theta_{i} & \cos \theta_{i}\end{array}\right]$

where $A_{i}$ is the amplitude, $x_{0 i}$ and $y_{0 i}$ are the centre coordinates, $\theta_{i}$ the anti-clockwise rotation angle, and $\sigma_{x i}$ and $\sigma_{y i}$ the width along major and minor axis of the $i$ th Gaussian ellipsoid. The fitted ellipses are summed up and indicated by white lines. $A_{N+1}$ is the background brightness. The number $N$ is determined by the number of peaks above a certain threshold.

The Doppler velocity is shown in the middle row of Fig. 4. The radial velocity becomes larger with increasing distance from the origin along the wind direction. This is reasonable with a horizontal wind component, given that the projected radial velocity depends on the off-zenith angle. The increase in radial velocity is not steady, indicating wind variability within the observed area. The belt of positive radial velocity that can be seen in all three images might be caused by spikes in the spectrum due to low SNR.

The spectral width is also not uniform (Fig. 4, bottom). Areas with increased brightness show a small spectral width while areas with increased spectral width occur mostly at larger distances from zenith, leading to an apparent larger spectral width.

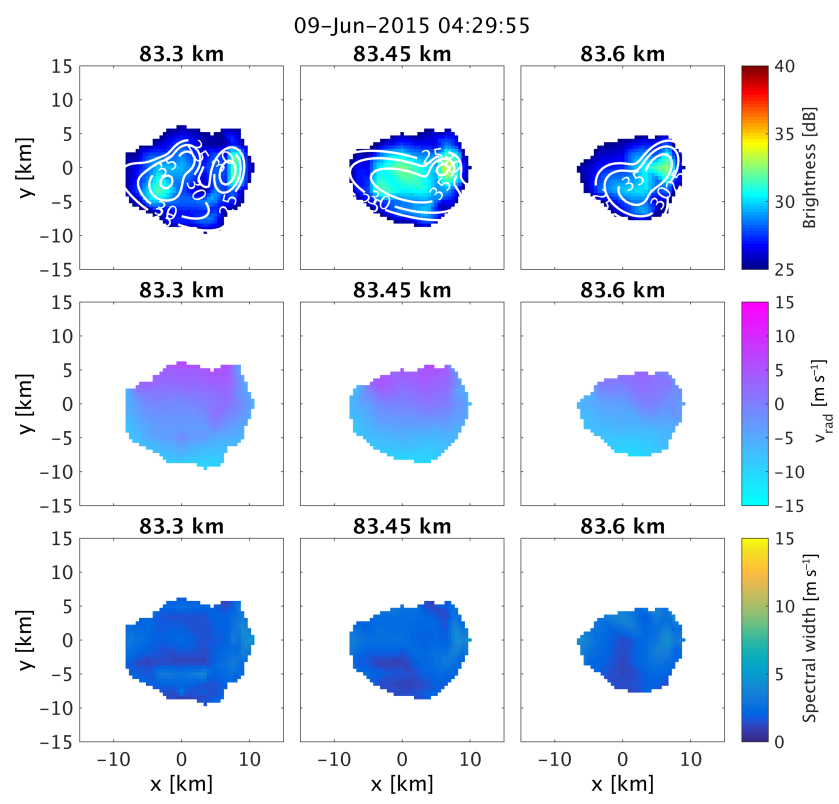

Figure 5. Same as Fig. 4 but for 04:29:55 UTC and around $83.45 \mathrm{~km}$.

A second example of PMSE images is shown in Fig. 5. It is similar to Fig. 4 but at 04:29:55 UTC. In contrast to Fig. 4, PMSEs are weaker and the beam volume is not completely filled. The radial velocities also indicate a horizontal wind field but show fewer variations than the wind field shown in Fig. 5. The spectral width maps do not show increased spectral width.

Furthermore, as in Fig. 4, the brightness distribution in Fig. 5 is neither homogeneous nor similar to the antenna beam pattern. Again, the fitted brightness peaks are indicated by white lines and several peaks can be identified at one altitude. Although the brightness distribution is influenced by the antenna beam pattern, the patches of brightness allow us to determine the observed patch size by radar. To compare the different observation volumes, the fitted widths $\sigma_{x}$, $\sigma_{y}$ and centres $x_{0}, y_{0}$ of all Gaussian ellipsoids fitted in the time period shown in Fig. 3, and for all altitudes containing PMSEs, are shown in Fig. 6. Although a similar approach was shown by Chilson et al. (2002) and Chen et al. (2008), our imaging configuration, i.e. longer and higher number of unique baselines than previously used, allows the detection of several patches and we interpret our results as patches and not as aspect sensitivity as Chen et al. (2008) had done. Furthermore, we use different beam sizes and integration times given that Sommer et al. (2016b) showed that longer integration times lead to a more homogeneously filled beam volume. The width is shown in the first row for $x$ (left) and $y$ (right), and the count number for each bin is normalized to the total count number. Colour coded are the different beam sizes and integration times. Yellow indicates the $3.6^{\circ}$ narrow beam with $32 \mathrm{~s}$ integration time. The $12.6^{\circ}$ wide beam is shown in 

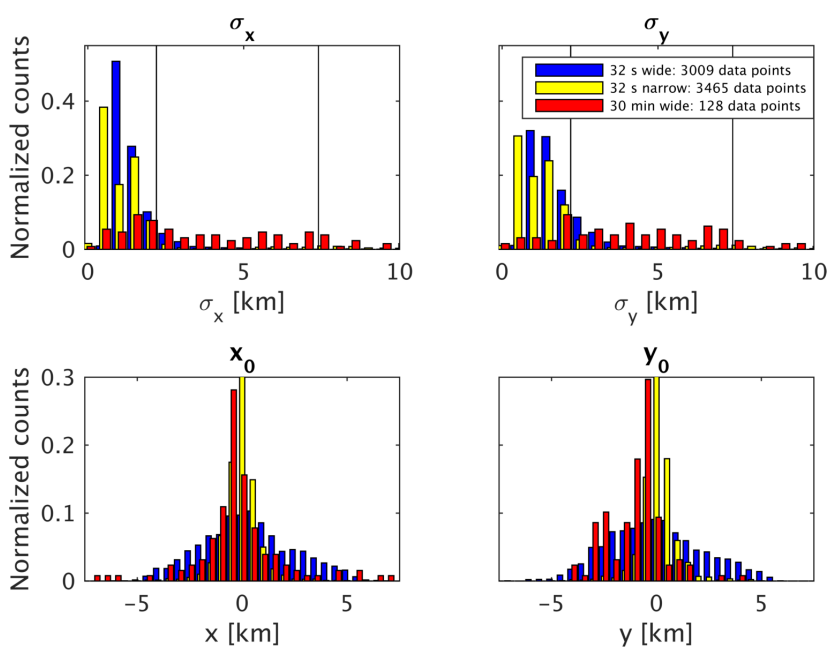

Figure 6. Histograms of the widths in $x$ and $y$ direction (top row) and centre locations (bottom row). For each figure, three histograms are shown. Yellow: $32 \mathrm{~s}$ narrow beam, blue: $32 \mathrm{~s}$ wide beam, red: 30 min wide beam

blue for $32 \mathrm{~s}$ integration period and in red for $30 \mathrm{~min}$ by averaging the spectra of the $32 \mathrm{~s}$ data set. The two black vertical lines indicate the $3 \mathrm{~dB}$ beam sizes at $85 \mathrm{~km}$ for the narrow $(2.2 \mathrm{~km})$ and wide beam $(7.4 \mathrm{~km})$, respectively. The bottom row shows the centre locations in $x$ and $y$ direction.

The patch sizes of the $32 \mathrm{~s}$ narrow and wide beams are rather similar with the peak of the size distribution under $2 \mathrm{~km}$ and therefore smaller than the narrow main beam in $x$ as well $y$ direction. The wide beam patch distribution shows a shift to slightly larger values than the narrow beam patch distribution in both directions. This is probably due to the antenna beam pattern influence. The $30 \mathrm{~min}$ wide beam distribution does not show a distinct peak but a rather wide distribution of patch sizes. This wider distribution might result from a non-homogeneous antenna beam pattern and imaging instrument function (see Fig. 2, right panel), as the Gaussian ellipsoid detection algorithm might detect only several distinctive peaks. The second row shows the centre locations. All distributions are almost centred around the zenith but show different widths. The $32 \mathrm{~s}$ narrow beam centre distributions in $x$ and $y$ direction have the smallest width as the centre location is limited by the antenna beam pattern. The $32 \mathrm{~s}$ wide beam centre distributions have the broadest width, as patches of PMSEs can occur in a larger beam volume, and hence the spread is larger than in the narrow beam centre distribution. On the other hand, the 30 min wide beam centre distributions are narrower than the $32 \mathrm{~s}$ distribution. With longer integration time, the antenna beam pattern should become more dominant and reduces the patchiness of PMSEs, resulting in a more centred distribution for the narrow beam.

\subsection{Enhanced spectral width}

The distinction between main beam and side lobe detection is crucial. Even with a strong side lobe attenuation of $-17 \mathrm{~dB}$ of the first side lobe for its standard narrow beam, MAARSY is able to receive significant contributions of signals from the side lobes when strong PMSEs occur. Therefore, we show a way to identify the side lobe signals and improve the estimates of radial velocity and spectral width.

To identify main beam and side lobe detections, we apply radar imaging as described above, resulting in a spectrum for each virtual beam pointing direction $\boldsymbol{k}$. Each spectrum was analysed regarding Doppler velocity and spectral width.

In order to avoid large angle contributions, we use imaging and compare the results to the standard narrow beam, for which we assume that all echoes come from the main beam. We averaged the spectra, obtained from imaging, only for a certain area, namely between -1.8 and $1.8^{\circ}$, hereafter called synthetic narrow beam, and determined the spectral width from the average of the spectra. The area corresponds to the HPFW main beam of MAARSY. Figure 7 shows range time intensity plots (RTIs) of the SNR and spectral width for the standard narrow beam (left column) and the synthetic narrow beam (right column). It can be seen that the spectral width of the standard narrow beam shows sometimes an increase at the upper edge of PMSEs. These features vanishes when the spectrum is only obtained from the synthesized narrow beam. This can be clearly seen around 06:20 UTC around $85 \mathrm{~km}$. However, there are periods with increased spectral width in the synthetic narrow beam, possibly related to increased turbulence, e.g. around 08:00 UTC.

We used the approach of Hocking (1985) to estimate the turbulence strength in a simple approach by neglecting shear and wave broadening (Murphy et al., 1994; Nastrom and Eaton, 1997). We estimated the horizontal wind velocity using the derived radial velocity maps presented above using a DBS approach. The resulting wind magnitude of the horizontal wind is presented in Fig. 8, top. Throughout the observation period, increased periods of wind can be detected, leading to an increased spectral width due to beam broadening. Following Hocking (1985), the turbulence strength $\epsilon$ can be derived from the HPFW of the spectrum by

$f_{\text {turb }}^{2}=f_{\text {obs }}^{2}-f_{\text {bb }}^{2}$,

where $f_{\text {turb }}$ is the increase in spectral width due to turbulence, $f_{\mathrm{obs}}$ is the measured HPFW of the spectrum and $f_{\mathrm{bb}}=$ $\frac{2}{\lambda} f_{3 \mathrm{~dB}} V$ the increase in spectral width due to a horizontal wind $V$ (i.e. so called beam broadening effect), calculated by using the $3 \mathrm{~dB}$ half width beam size. The two-way beam width for the narrow beam of MAARSY is $f_{3 \mathrm{~dB}}=1.3^{\circ}$. Theoretically, the two-way beam width for the narrow beam, and therefore the beam broadening effect, would increase if the side lobe contributions were significant. We calculated $f_{3 \mathrm{~dB}}=0.95^{\circ}$ for the synthetic narrow beam with the second moment and assuming the standard narrow beam shape, 

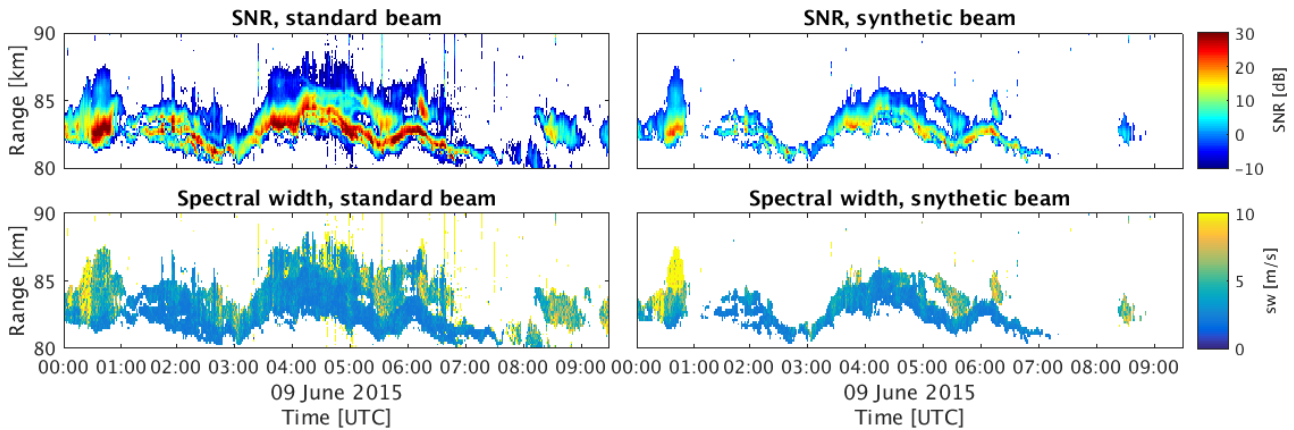

Spectral width, snythetic beam

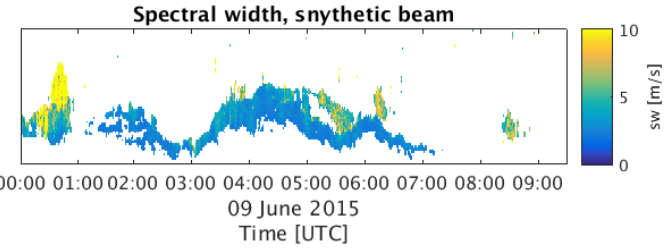

Figure 7. Comparison of spectral parameters obtained from the standard narrow beam, including side lobes (left column), and the synthetic narrow beam (right column). The synthetic narrow beam was obtained by gaining spectra for each virtual beam pointing direction $\boldsymbol{k}$ and average the resulting spectra only between -1.8 and $1.8^{\circ}$. Comparing the SNR (first row), the echoes from the upper edges of PMSEs are removed in the main beam while the lower edges are in the same range. Signals with large spectral width (second row) due to side lobe detections are removed from the beam and increased spectral width to turbulence remains.

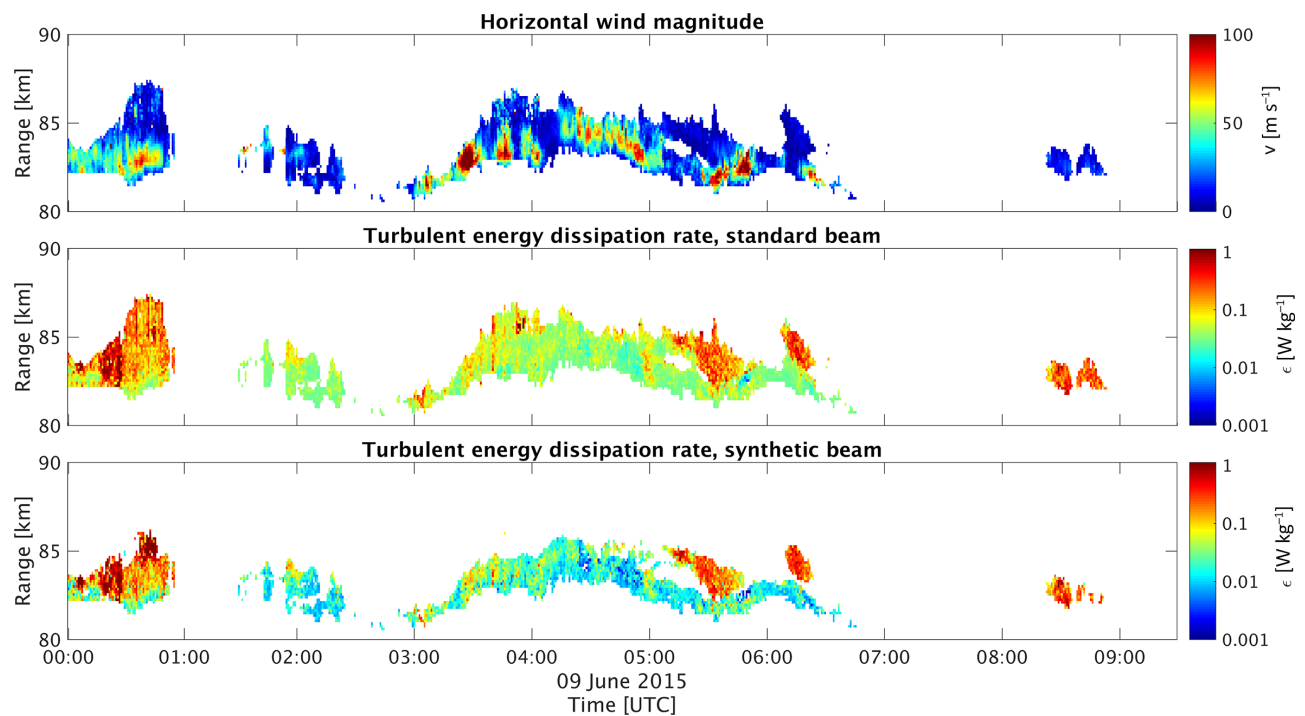

Figure 8. Top: magnitude of the horizontal wind velocity derived by DBS from Doppler velocity maps. Middle: derived energy dissipation rate for the standard narrow beam. Bottom: derived turbulent energy dissipation rate for a synthetic narrow beam.

which, however, is zero outside the MAARSY main beam (i.e. $>1.8^{\circ}$ ).

The mean square fluctuation velocity $v_{\mathrm{rms}}^{2}$ is given by

$v_{\mathrm{rms}}^{2}=\frac{\lambda^{2}}{4} \frac{f_{\mathrm{turb}}^{2}}{2 \ln 2}$

yielding for $\epsilon$ :

$\epsilon=C N v_{\mathrm{rms}}^{2}$,

where $C$ is a numerical constant and $N$ the Brunt-Väisälafrequency. Here, we use typical values for the polar mesosphere, i.e. $C=0.47$ and $N=0.0134 \mathrm{rad} \mathrm{s}^{-1}$ (Gibson-Wilde et al., 2000). The results for $\epsilon$ are presented in Fig. 8, middle, for the standard narrow beam, including side lobe contributions, and in the bottom panel for the synthetic narrow beam after removing the side lobe contributions. The observed spectral widths for the standard narrow beam are usually larger than for the synthetic narrow beam, and hence the energy dissipation rates for the synthetic narrow beam are smaller. The turbulence strength varies in both the narrow beam with side lobe contribution and the synthetic narrow beam without side lobe contribution, with increased turbulence strength at some parts of PMSEs, especially around 00:00-01:00, 03:00-07:00 for some parts and 08:30-09:00, with $\epsilon>500 \mathrm{~mW} \mathrm{~kg}^{-1}$. Identifying the side lobes leads to a decrease in energy dissipation rate. This can be seen in Fig. 9, where the energy dissipation rate is plotted as a 2-D histogram (upper left panel), especially for small $\epsilon$, where the 2-D correlation deviates from the line of equality. The upper right and lower panel shows the cumulative histograms in blue for the synthetic narrow and red for the standard narrow 

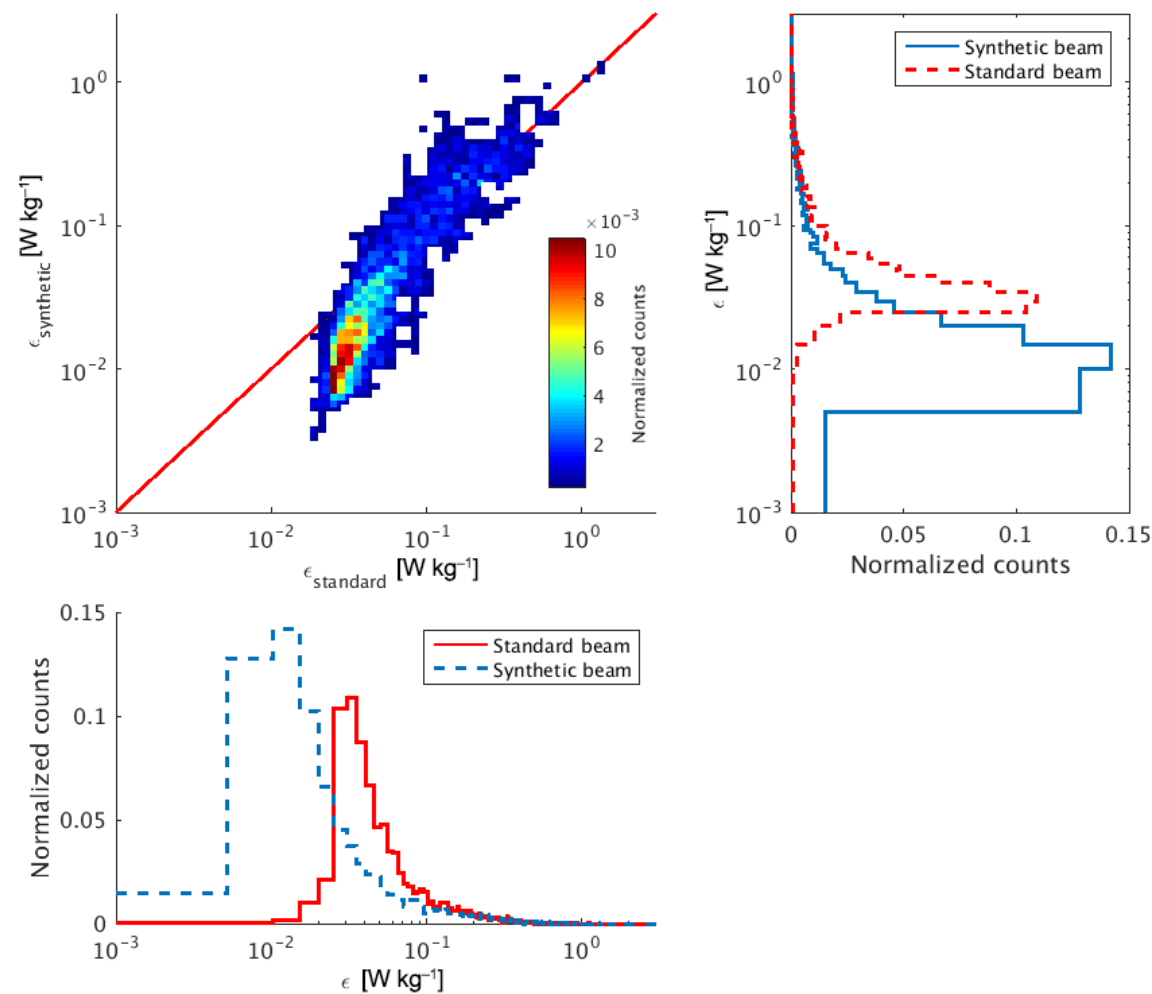

Figure 9. Histogram for the energy dissipation rate for $\epsilon$ derived by the narrow beam and the synthetic narrow beam. Top left: 2-D correlation in $\epsilon$ between the standard narrow beam and the synthetic narrow beam. The red line indicates $x=y$. A deviation can be seen especially for small $\epsilon$. The cumulative histograms along $x$ and $y$ direction are shown in the top right and lower panels, respectively. The red line shows the cumulative histogram for the standard narrow beam and the blue line for the synthetic narrow beam.

beam. For better comparison, the other histogram is shown with a dashed line. For low energy dissipation rates, comparing the standard narrow beam and the synthetic narrow beam, a shift towards lower energy dissipations rates can be seen here also. That means that correcting for side lobe contribution affects mainly low-turbulence cases.

\subsubsection{Discussion}

The horizontal variation on larger scales have been investigated by multi-beam experiments (Latteck et al., 2012; Stober et al., 2013). They showed that PMSEs can vary within observation volumes of $80 \mathrm{~km}$ diameter by $40 \mathrm{~dB}$ in SNR. Multi-beam experiments take time and the resolution is limited by the beam size. Röttger et al. (1990) concluded from spectral observations that PMSEs at $224 \mathrm{MHz}$ must be smaller than their observation volume, i.e. $1 \mathrm{~km}$ in vertical and horizontal extent. To investigate the horizontal structure of PMSEs further and quantify the localized scattering, we applied Capon's method of imaging. As shown in the results section above, PMSEs vary in altitude, horizontal location and extent. PMSEs are composed of isotropic scattering organized in horizontally contiguous areas. Sometimes, the beam volume is filled completely with isotropic scattering, but the angular power distribution is not homogeneous
(Fig. 4). In other cases, PMSEs appear in patches of isotropic scattering that are asymmetric and can be smaller than $1 \mathrm{~km}$. Even with the narrow beam experiment with a beam width of $3.6^{\circ}$, the brightness distribution within the observation volume is not homogeneous. It can be seen in Fig. 6 that the narrow and wide beam patches for $32 \mathrm{~s}$ integration time are of the same order of magnitude, although one would expect that the antenna beam pattern has a major influence. This might be due to the receiving antenna pattern, which is limited by the receiver configuration (compare to Fig. 1). Furthermore, the centre location of especially the wide beam patches is slightly shifted towards negative $y_{0}$. This is probably due to a small phase calibration offset but the main features of PMSEs are preserved.

The hypothesis that PMSEs are non-homogeneous and sometimes localized was stated in Röttger et al. (1990) at $224 \mathrm{MHz}$ and in Sommer et al. (2016b) at 53.5 MHz but without imaging. Here, we can support that statement and furthermore also show that PMSEs were composed of localized structures of a few kilometres. If PMSEs were highly aspectsensitive, imaging of wide beam experiments would show an increased brightness around zenith and almost no scatter at larger off-zenith angles. In our images, we do not see this; the images are only weighted by the antenna gain. Therefore the 
scattering process should be isotropic. This is similar to Chen et al. (2008), who studied mesospheric echoes using imaging with the OSWIN radar and find also sometimes several backscatter locations. They interpreted their results as several reflection points from wave structures and not as isotropic scattering. Yu et al. (2001) showed also two different scattering locations in CRI images of PMSEs and used them to explain frequency jumps in PMSE observations. Chilson et al. (2002) used also CRI to estimate the observed CRI patch size and showed that it was smaller than the beam volume and interpreted this as aspect-sensitive scattering. However, the resolution of the radars used in these studies is lower than the resolution presented in this paper and smaller structures might not have been resolved. Furthermore, we showed that PMSE backscatter can be received from the whole beam volume, which makes it unlikely to be aspect-sensitive scattering. Still, the structure of the isotropic scattering can be influenced by gravity waves as suggested by Chen et al. (2008) for the reflection type of scattering.

The inhomogeneous structure is due to the nature of PMSEs. As Rapp and Lübken (2004) pointed out, three major components must be present for PMSEs to exist: negatively charged ice particles, free electrons and turbulence. Baumgarten and Fritts (2014, Fig. 2) showed in NLC observations that ice particles in mesospheric altitudes also show wave structures with short wavelengths $(<20 \mathrm{~km})$ when ice particles are moved to different altitudes. Hence, it is not surprising that PMSEs, bound to the existence of these ice particles, display also wave structures on the small scale.

In addition to the patchy structure, we observe enhanced brightness within the observation volume, when PMSEs fill the complete beam volume. This might be caused by localized enhanced turbulence or electron density but needs further investigation.

The non-homogeneous PMSE distribution in space has effects on measurement techniques for wind and/or turbulence estimations such as DBS or FCA. FCA is, as an in-beam estimation method, especially influenced by small-scale wave activity, as the ground diffraction pattern is used to determine atmospheric parameters. Usually, the derivation required a statistically homogeneous scatter distribution with a vertical anisotropy (Doviak et al., 1996) or additional anisotropy in $x$ and $y$ direction (Holloway et al., 1997). In addition to the anisotropy of the scattering mechanism, the scatter itself might not be statistically homogeneously distributed in the observation volume, which can lead to an underestimation of the horizontal wind velocity (Holdsworth, 1995). This might be due to localized enhancements, patches or waves. Usually, the sampling time used for FCA is about $30 \mathrm{~s}$ as used in the results presented here. We showed that, on these timescales, the distribution of PMSEs in the beam volume is not homogeneous. The non-homogeneity leads to an increased correlation compared to a statistically homogeneous scattering process and was previously interpreted as aspect sensitivity. Sommer et al. (2016b) compared aspect sensitivity values ob- tained from multiple beam experiments to values obtained by a spatial correlation analysis and concluded that the DBS method requires long integration time for aspect sensitivity measurements and that the enhanced correlation values on short timescales are due to localized scattering mechanisms. Here, we can support this hypothesis by showing the nonhomogeneity of PMSEs on the $30 \mathrm{~s}$ timescale in the observation volume.

To study PMSEs with different radar systems, the volume reflectivity is commonly used (Hocking, 1985; Li et al., 2010; Smirnova et al., 2011; Latteck and Bremer, 2013). The assumption is that the scattering mechanism is isotropic and also homogeneous. We have shown above that on short timescales the assumption of a homogeneous scattering process is not necessarily given, resulting in a smaller volume reflectivity factor. This can be solved by calculating a beamfilling factor for the volume reflectivity or, following the approach of Sommer et al. (2016b), by using longer integration periods. Latteck and Bremer (2013) used integration times of $5 \mathrm{~min}$, which smoothes the localized signals and is already 10 times longer than the data sets presented here, while even longer integration periods would be more favourable.

In the second part of the discussion, we discuss radar imaging to remove side lobe detections. Mesospherestratosphere-troposphere (MST) radars like MAARSY are used to study atmospheric parameters such as radial velocities for wind estimations and spectral width for turbulence estimations. Sensitive radar systems have a good side lobe suppression (e.g. MAARSY $-17 \mathrm{~dB}$ one way; Latteck et al., 2012). The suppression of MAARSY is better than older systems like ALWIN (Alomar wind radar) $(-13 \mathrm{~dB}$ one way, Latteck et al., 1999), but MAARSY still receives significant backscatter from the side lobes. If these side lobe detections are not separated from the main beam detections, the results are compromised. In this paper, we showed that, with the help of imaging, side lobe detections of PMSEs could be reduced significantly. The cleaned spectrum, only for the main beam, can now be analysed regarding the spectral parameters. As shown above, the side lobe detections have a major influence on the spectral width and therefore on turbulence estimations. On the other hand, we can use the information from the side lobes with imaging to resolve the spectral width and Doppler velocity in space as shown in Sect. 2.2.

Although many PMSEs with apparent large spectral width could be identified as side lobe contributions, sometimes the spectral width of the remaining PMSEs is enhanced, indicating increased turbulence or beam broadening due to increased horizontal winds or other effects (Hocking, 1985). We estimated the turbulence strength from a synthetic narrow beam of $3.6^{\circ}$ with imaging as well as the standard narrow beam. Different derived turbulence strengths in PMSEs can be identified. The derived turbulence strength $\epsilon$ is especially in the lower part of PMSEs rather small with $\epsilon \sim 10 \mathrm{~mW} \mathrm{~kg}^{-1}$. This is of the order of expected values of turbulence in the mesopause region. Rapp and Lübken 
(2004) expected $\epsilon=5 \mathrm{~mW} \mathrm{~kg}^{-1}$, while Gibson-Wilde et al. (2000) simulated values up to $\epsilon=150 \mathrm{~mW} \mathrm{~kg}^{-1}$ using a direct numerical simulation. Li et al. (2010) found energy dissipation rates using the European Incoherent Scatter Svalbard Radar at $500 \mathrm{MHz}$ (Bragg wavelength $30 \mathrm{~cm}$ ) of $\epsilon=5-200 \mathrm{~mW} \mathrm{~kg}^{-1}$. Our observations agree also well with in situ measurements. Sounding rocket flights conducted by Lübken et al. (2002) measured values between $\epsilon=0 \mathrm{~mW} \mathrm{~kg}^{-1}$ and $\epsilon \sim 2400 \mathrm{~mW} \mathrm{~kg}^{-1}$ in the mesosphere. The mean value where PMSEs and turbulence coincided in the flights was $\epsilon=390 \mathrm{~mW} \mathrm{~kg}^{-1}$ with a rather large standard deviation of $190 \mathrm{~mW} \mathrm{~kg}^{-1}$.

We also found strong turbulent events within PMSEs, even after removing side lobe contribution and beam broadening. Strong turbulent events showed $\epsilon>500 \mathrm{~mW} \mathrm{~kg}^{-1}$, which exceeds the expected theoretical values but still agrees with the sounding rocket measurements of Lübken et al. (2002). Furthermore, we showed that the corrections made in this paper affect the majority of low-turbulence cases. For high turbulence values, the synthetic narrow beam and the standard narrow beam values correlate well.

In this calculation, we neglected shear broadening as the range resolution is high and the shear contribution probably small compared to the other effects (Strelnikova and Rapp, 2011). We neglected also wave broadening, which would allow for high-frequency gravity waves. Still, the observed spectral width should exceed the contribution of shear and wave broadening and is therefore an indicator of strong turbulence. The analysis presented here should be expanded in future to include shear and beam broadening effects, which might have a significant contribution in weak turbulence measurements.

\section{Conclusions}

In this paper, we showed that PMSEs appear sometimes in horizontally contiguous areas, or patches, smaller than even $1 \mathrm{~km}$, usually in patches with a few kilometres in diameter, and sporadically comparable in size to the observed volume ( $\sim 20 \mathrm{~km}$ ) using radar imaging. Large patches of PMSEs can be observed on some occasions, but these patches are not homogeneous. These inhomogeneities can be explained by an isotropic scattering mechanism that is probably influenced by the background dynamics, creating the patchiness. Long integration periods could be used to smooth out the patchy nature of PMSEs for experiments that do not require a high temporal resolution. The patchy structure of PMSEs might be misinterpreted as aspect sensitivity, which should be considered in future investigations of PMSEs.

Furthermore, we showed that radar imaging can be used to identify side lobe contributions in spectral width that occur even in modern radar systems like MAARSY. The method presented here can be used to improve turbulence measurements with MST radars. We found that the correction is sig- nificant most of the time in the analysed data. Events characterized by high spectral width show similar turbulence values before and after the beam broadening corrections.

\section{Data availability}

The raw data are not openly available since they are being used for other research topics. However, those interested can contact Jorge Chau (chau@iap-kborn.de) to get limited access to the data.

Acknowledgements. The authors thank the MAARSY team, especially Ralph Latteck for his support during the imaging campaign, Toralf Renkwitz for providing the wide beam phases and also Bjørn Gustavsson from University of Troms $\emptyset$ for his help choosing the imaging configuration. We also thank Gunter Stober for providing the spectral fit algorithm used in the imaging analysis. Svenja Sommer was supported by ILWAO.

The topical editor, C. Jacobi, thanks two anonymous referees for their help in evaluating this paper.

\section{References}

Baumgarten, G. and Fritts, D. C.: Quantifying Kelvin-Helmholtz instability dynamics observed in noctilucent clouds: 1. Methods and observations, J. Geophys. Res., 119, 9324-9337, doi:10.1002/2014JD021832, 2014.

Briggs, B. H.: On the analysis of moving patterns in geophysics- I. Correlation analysis, J. Atmos. Terr. Phys., 30, 1777-1788, 1968.

Capon, J.: High-Resolution Frequency-Wavenumber Spectrum Analysis, IEEE Proc., 57, 1408-1418, 1969.

Chau, J. L. and Woodman, R. F.: Three-dimensional coherent radar imaging at Jicamarca: comparison of different inversion techniques, J. Atmos. Sol.-Terr. Phy., 63, 253-261, 2001.

Chen, J.-S., Hoffmann, P., Zecha, M., and Hsieh, C.-H.: Coherent radar imaging of mesosphere summer echoes: Influence of radar beam pattern and tilted structures on atmospheric echo center, Radio Sci., 43, RS1002, doi:10.1029/2006RS003593, 2008.

Chilson, P. B., Yu, T.-Y., Palmer, R. D., and Kirkwood, S.: Aspect sensitivity measurements of polar mesosphere summer echoes using coherent radar imaging, Ann. Geophys., 20, 213-223, doi:10.5194/angeo-20-213-2002, 2002.

Czechowsky, P., Reid, I. M., Rüster, R., and Schmidt, G.: VHF Radar Echoes Observed in the Siummer and Winter Polar Mesosphere Over Andøya, Norway, J. Geophys. Res., 94, 5199-5217, 1989.

Doviak, R. J., Lataitis, R. J., and Holloway, C. L.: Cross correlations and cross spectra for spaced antenna wind profilers: 1 . Theoretical analysis, Radio Sci., 31, 157-180, 1996.

Gibson-Wilde, D., Werne, J., Fritts, D., and Hill, R.: Direct numerical simulation of VHF radar measurements of turbulence in the mesosphere, Radio Sci., 35, 783-798, doi:10.1029/1999RS002269, 2000.

Hocking, W. K.: Measurement of turbulent energy dissipation rates in the middle atmosphere by radar techniques: A review, Radio Sci., 20, 1403-1422, 1985. 
Hocking, W. K., Ruster, R., and Czechowsky, P.: Absolute reflectivities and aspect sensitivities of VHF radiowave scatterers measured with the SOUSY radar, J. Atmos. Terr. Phys., 48, 131-144, 1986.

Holdsworth, D. A.: Signal analysis with applications to atmospheric radars, $\mathrm{PhD}$ thesis, University of Adelaide, 1995.

Holloway, Christopher L., Doviak, R. J., and Cohn, S. A.: Cross correlations of fields scattered by horizontally anisotropic refractive index irregularities, Radio Sci., 32, 1911-1920, doi:10.1029/97RS00715, 1997.

Hoppe, U.-P. and Fritts, D. C.: High-resolution measurements of vertical velocity with the European incoherent scatter VHF radar 1. Motion field characteristics and measurement biases, J. Geophys. Res., 100, 16813-16825, 1995.

Hysell, D. L. and Chau, J. L.: Optimal aperture synthesis radar imaging, Radio Sci., 41, 1-12, doi:10.1029/2005RS003383, 2006.

Kelley, M., Ulwick, J., Röttger, J., Inhester, B., Hall, T., and Blix, T.: Intense turbulence in the polar mesosphere: rocket and radar measurements, J. Atmos. Terr. Phys., 52, 875-891, doi:10.1016/0021-9169(90)90022-F, 1990.

Kudeki, E. and Sürücü, F.: Radar interferometric imaging of fieldaligned plasma irregularities in the equatorial electrojet, Geophys. Res. Lett, 18, 41-44, doi:10.1029/90GL02603, 1991.

Latteck, R. and Bremer, J.: Long-term changes of polar mesosphere summer echoes at $69^{\circ} \mathrm{N}$, J. Geophys. Res., 118, 10441-10448, doi:10.1002/jgrd.50787, 2013.

Latteck, R., Singer, W., and Bardey, H.: The ALWIN MST Radar: Technical Design and Performance, European Rocket and Balloon Programs and Related Research, Proceedings of the 14th ESA Symposium held 31 May-3 June, 1999, Potsdam, edited by: Schürmann, B., European Space Agency, ESA-SP, 437, 179184, 1999.

Latteck, R., Singer, W., Rapp, M., Vandepeer, B., Renkwitz, T., Zecha, M., and Stober, G.: MAARSY: The new MST radar on Andøya-System description and first results, Radio Sci., 47, RS1006, doi:10.1029/2011RS004775, 2012.

Li, Q., Rapp, M., Röttger, J., Latteck, R., Zecha, M., Strelnikova, I., Baumgarten, G., Hervig, M., Hall, C., and Tsutsumi, M.: Microphysical parameters of mesospheric ice clouds derived from calibrated observations of polar mesosphere summer echoes at Bragg wavelengths of $2.8 \mathrm{~m}$ and $30 \mathrm{~cm}$, J. Geophys. Res., 115, D00I13, doi:10.1029/2009JD012271, 2010.

Lübken, F.-J., Rapp, M., and Hoffmann, P.: Neutral air turbulence and temperatures in the vicinity of polar mesosphere summer echoes, J. Geophys. Res., 107, 4273, doi:10.1029/2001JD000915, 2002.

Murphy, D., Hocking, W., and Fritts, D.: An assessment of the effect of gravity waves on the width of radar Doppler spectra, J. Atmos. Terr. Phys., 56, 17-29, doi:10.1016/0021-9169(94)901724, 1994.
Nastrom, G. D. and Eaton, F. D.: Turbulence eddy dissipation rates from radar observations at $5-20 \mathrm{~km}$ at White Sands Missile Range, New Mexico, J. Geophys. Res., 102, 19495-19505, 1997.

Palmer, R. D., Gopalam, S., Yu, T. Y., and Fukao, S.: Coherent radar imaging using Capon's method, Radio Sci., 33, 1585-1598, 1998.

Rapp, M. and Lübken, F.-J.: Polar mesosphere summer echoes (PMSE): Review of observations and current understanding, Atmos. Chem. Phys., 4, 2601-2633, doi:10.5194/acp-4-2601-2004, 2004.

Röttger, J., Rietveld, M. T., La Hoz, C., Hall, T., Kelley, M. C., and Swartz, W. E.: Polar mesosphere summer echoes observed with the EISCAT 933-MHz radar and the CUPRI 46.9-MHz radar, their similarity to $224-\mathrm{MHz}$ radar echoes, and their relation to turbulence and electron density profiles, Radio Sci., 25, 671-687, doi:10.1029/RS025i004p00671, 1990.

Sheth, R., Kudeki, E., Lehmacher, G., Sarango, M., Woodman, R., Chau, J., Guo, L., and Reyes, P.: A high-resolution study of mesospheric fine structure with the Jicamarca MST radar, Ann. Geophys., 24, 1281-1293, doi:10.5194/angeo-24-12812006, 2006.

Smirnova, M., Belova, E., and Kirkwood, S.: Polar mesosphere summer echo strength in relation to solar variability and geomagnetic activity during 1997-2009, Ann. Geophys., 29, 563-572, doi:10.5194/angeo-29-563-2011, 2011.

Sommer, S., Chau, J. L., and Schult, C.: On high time-range resolution observations of PMSE: statistical characteristics, J. Geophys. Res., 121, 6713-6722, doi:10.1002/2015JD024531, 2016a

Sommer, S., Stober, G., and Chau, J. L.: On the angular dependence and scattering model of Polar Mesospheric Summer Echoes at VHF, J. Geophys. Res., 121, 278-288, doi:10.1002/2015JD023518, 2016b.

Stober, G., Sommer, S., Rapp, M., and Latteck, R.: Investigation of gravity waves using horizontally resolved radial velocity measurements, Atmos. Meas. Tech., 6, 2893-2905, doi:10.5194/amt6-2893-2013, 2013.

Strelnikova, I. and Rapp, M.: Majority of PMSE spectral width at UHF and VHF are compatible with a single scattering mechanism, J. Atmos. Sol.-Terr. Phy., 73, 2142-2152, 2011.

Waldteufel, P. and Corbin, H.: On the analysis of single Doppler data, J. Appl. Meteorol., 18, 53-542, 1979.

Yu, T. Y., Palmer, R. D., and Hysell, D. L.: A simulation study of coherent radar imaging, Radio Sci., 35, 1129-1141, 2000.

Yu, T.-Y., Palmer, R. D., and Chilson, P. B.: An investigation of scattering mechanisms and dynamics on PMSE using coherent radar imaging, J. Atmos. Sol.-Terr. Phy., 63, 1797-1810, 2001.

Zecha, M., Röttger, J., Singer, W., Hoffmann, P., and Keuer, D.: Scattering properties of PMSE irregularities and refinement of velocity estimates, J. Atmos. Sol.-Terr. Phy., 63, 201-214, 2001. 